\title{
Resolution Enhancement for Drill-Core Hyperspectral Mineral Mapping
}

\author{
Isabel Cecilia Contreras Acosta ${ }^{1,2, *(\mathbb{D})}$, Mahdi Khodadadzadeh ${ }^{1,3}\left(\mathbb{D}\right.$ and Richard Gloaguen ${ }^{1}$ (D) \\ 1 Helmholtz-Zentrum Dresden-Rossendorf, Helmholtz Institute Freiberg for Resource Technology, \\ Chemnitzer Str. 40, 09599 Freiberg, Germany; m.khodadadzadeh@utwente.nl (M.K.); \\ r.gloaguen@hzdr.de (R.G.) \\ 2 TheiaX, HZDR Innovation GmbH, Bautzner Landstraße 400, 01328 Dresden, Germany \\ 3 Faculty of Geo-Information Science and Earth Observation (ITC), University of Twente, \\ 7500 AE Enschede, The Netherlands \\ * Correspondence: i.contreras@hzdr.de or c.contreras@hzdri.de; Tel.: +49-351-260-4743
}

Citation: Contreras Acosta, I.C.; Khodadadzadeh, M.; Gloaguen, R. Resolution Enhancement for Drill-Core Hyperspectral Mineral Mapping. Remote Sens. 2021, 13, 2296. https://doi.org/10.3390/rs13122296

Academic Editor: Lenio Soares Galvao

Received: 23 April 2021

Accepted: 8 June 2021

Published: 11 June 2021

Publisher's Note: MDPI stays neutra with regard to jurisdictional claims in published maps and institutional affiliations.

Copyright: (c) 2021 by the authors. Licensee MDPI, Basel, Switzerland. This article is an open access article distributed under the terms and conditions of the Creative Commons Attribution (CC BY) license (https:// creativecommons.org/licenses/by/ $4.0 /)$
Abstract: Drill-core samples are a key component in mineral exploration campaigns, and their rapid and objective analysis is becoming increasingly important. Hyperspectral imaging of drill-cores is a non-destructive technique that allows for non-invasive and fast mapping of mineral phases and alteration patterns. The use of adapted machine learning techniques such as supervised learning algorithms allows for a robust and accurate analysis of drill-core hyperspectral data. One of the remaining challenge is the spatial sampling of hyperspectral sensors in operational conditions, which does not allow us to render the textural and mineral diversity that is required to map minerals with low abundances and fine structures such as veins and faults. In this work, we propose a methodology in which we implement a resolution enhancement technique, a coupled non-negative matrix factorization, using hyperspectral, RGB images and high-resolution mineralogical data to produce mineral maps at higher spatial resolutions and to improve the mapping of minerals. The results demonstrate that the enhanced maps not only provide better details in the alteration patterns such as veins but also allow for mapping minerals that were previously hidden in the hyperspectral data due to its low spatial sampling.

Keywords: resolution enhancement; sampling enhancement; hyperspectral; high-spatial resolution multi-spectral; drill-cores; mineral mapping; machine learning

\section{Introduction}

Drilling is a crucial component of the discovery roadmap to identifying economically profitable ore deposits. Drill-cores are cylindrical rock samples drilled to depths that can reach several hundreds to a few thousand meters from the Earth's crust. They provide valuable information useful for characterizing geological ore deposits (e.g., geology and geological history of the deposits), aquifers, and geothermal prospects. Even if the overall budget for exploration and exploration drilling has decreased in the mining industry in the last two years due to economic reasons [1], there is an increasing need for more advanced and robust analyses of new and archived drill-cores. Indeed, in order to achieve the 2050 energy goals, new renewable energy networks such as wind, solar, and geothermal power require substantial amounts of minerals such as cobalt and lithium as well as base metals including copper and aluminium, perhaps up to 3 billion tons per year [2]. This growing need is critical not only in terms of the new 2050 energy goals but also in light of the extremely high cost of obtaining new samples from boreholes. As a result, the work presented here is critical in terms of data management and cost-related optimization.

Drill-cores are primarily characterized using traditional core logging techniques. Geologists, mainly on site, carefully inspect and qualitatively describe the characteristics of the drill-cores such as the rock type, texture, mineralogical assemblages, alteration facies, 
structures, and ore-forming minerals. This is, however, a subjective and time-consuming task relying on the knowledge and expertise of geologists. Besides core logging, analytical methods are also performed at meter intervals to obtain bulk geochemistry or on small representative sections of the cores to derive detailed quantitative information on mineralogy and geochemistry. Optical microscopy, X-ray diffraction and fluorescence, and scanning electron microscopy (SEM) are common analytical techniques in the mining industry [3-6]. These techniques are destructive and costly but provide objective measurements that can be used to support mining companies in their decision-making.

Mineral spectroscopy and more specifically hyperspectral imaging (HSI) has become an innovative and pioneer technology for the analysis of drill-cores in the last decade. This technique provides the means for a non-invasive, non-destructive, and rapid characterization of entire drill-cores. Hyperspectral data are recorded in several tens to hundreds of narrow and contiguous spectral bands along the electromagnetic spectrum covering from the visible and near infrared (VNIR), short-wave infrared (SWIR) to the long-wave infrared (LWIR) spectra depending on the sensor. Due to the high spectral resolution, the recorded data allow users to construct a rich reflectance spectrum that can be used to identify minerals or mineral mixtures and to map their spatial distribution, changes in composition, and relative abundance.

The reflectance spectrum of minerals varies, amongst other reasons, due to the chemical composition, mixtures in the mineral assemblages, and grain sizes. Each mineral has a diagnostic response in different parts of the electromagnetic spectrum as a result of the fundamental electronic and vibrational processes of the different atoms and molecular bonds $[7,8]$. For instance, mica, clay, chlorites, epidote, amphiboles, alunite, and gypsum have good diagnostic responses to short-wave infrared (SWIR) spectra and moderate responses to long-wave infrared (LWIR) spectra. Feldspars, quartz, and carbonates are distinguishable in the LWIR; however, carbonates also have a good diagnostic response in the SWIR. For a more detailed description of the infrared active minerals, the readers are referred to $[7,9]$.

Drill-core hyperspectral data have been analyzed following well-established methods such as minimum wavelength mapping, band ratios, spectral distance measurements using reference libraries, endmember extraction, and unmixing [8,10-12]. Machine learning techniques have also been implemented for the analysis of drill-core hyperspectral data in recent years to ameliorate the automation of analyses and to provide more robust results, especially by using supervised methods [13-17]. However, supervised learning algorithms require reference data (i.e., training sets) that can be difficult to obtain for drill-cores since, for example, they are not usually labelled at the millimeter scale. In our previous work [14], we proposed an innovative strategy to exploit geochemical data from assays performed over sections of ca. $1 \mathrm{~m}$ length to train a model and to estimate the element abundance information of more than $300 \mathrm{~m}$ of drill-cores by means of a superpixel segmentation of the hyperspectral data. In another work in [13], we upscaled high-resolution mineralogical data (i.e., model mineralogy with a ground sampling distance (GSD) of $3 \mu \mathrm{m}$ ) obtained with a scanning electron microscope (SEM) system coupled with the Mineral Liberation Analysis software (MLA) from small sections of drill-core samples to the entire drill-core samples by training a supervised machine learning model and by classifying the hyperspectral data. Following a similar strategy, in [16], the authors used SEM-MLA data to retrieve quasiquantitative mineral abundances from hyperspectral data based on regressions. Although these methods enable us to accurately map minerals, their relative abundances, and general distribution in drill-core data, they do not entirely exploit the full synergetic potential of SEM-MLA and hyperspectral data fusion due to the large difference between the spatial samplings of these two datasets. SEM-MLA data have a spatial sampling in the micrometer scale whereas hyperspectral data have a lower spatial sampling ranging in the millimeter scale. Therefore, detailed mineralogical and structural information is lost.

HS sensors can theoretically record several parts of the electromagnetic spectrum; however, technological constraints extremely limit the operational resolution and areas of 
the spectrum that can be measured at a given integration time. These technical limitations are the reasons why spatial resolution is one of the most expensive and difficult parameter required to improve imaging systems. These shortcomings constitute the requirements for post processing solutions. In this work, we propose a multi-resolution fusion-based framework to integrate RGB images in the chain of hyperspectral mineral mapping. As a complementary and intermediate source of information, the high-spatial resolution RGB images (0.1 mm GSD) compensate for the large difference between the spatial resolution of SEM-MLA (3 $\mu \mathrm{m}$ GSD) and hyperspectral data (1.5 mm GSD). More concretely, we propose enhancing the spatial resolution of hyperspectral data by means of co-registered RGB images before the integration of SEM-MLA and hyperspectral data. A variety of resolution enhancement techniques have been developed to improve the spatial resolution of multispectral images based on the fusion with high-spatial resolution panchromatic images, so called pansharpening [18] during the last decade. Based on the previously developed pansharpening techniques, different resolution enhancement methods have also been proposed for hyperspectral data. These techniques can be divided into two main groups: pansharpening-based and subspace methods. The first category includes techniques such as subdividing the hyperspectral data in different regions and applying conventional pansharpening techniques to fuse the hyperspectral and high spatial resolution multispectral (or RGB) data for each region [19], or synthesizing a high-resolution image for each band in the hyperspectral data as a linear combination of the multispectral/RGB band images via linear regression [20]. The subspace methods exploit the inherent spectral characteristics of hyperspectral data via a subspace spanned by a set of spectral signatures of the materials, the so-called endmembers, and consider the principle of spectral unmixing [21-24].

In our proposed approach, we enhance the resolution of the drill-core hyperspectral data by fusing high spatial resolution RGB data from the same drill-core sample. More specifically, we propose using subspace-based resolution enhancement methods because they preserve the spectral component in fusion better in comparison to pansharpeningbased methods, and this is highly relevant for mineral mapping [22]. This resolution enhancement allows for mapping minerals in drill-core hyperspectral data at a higher spatial resolution than traditional hyperspectral mineral mapping techniques. This enhancement fills the gap between the spatial resolution of detailed analytical analyses and hyperspectral data and, therefore, improves the identification of minerals and structural patterns in hyperspectral data that are hidden at the original resolution to a large extent. Moreover, highly detailed maps are important in the mining industry because only small portions of the drill-cores contain relevant mineralizations and vein structures. After enhancement of the hyperspectral sampling, we generate the reference data. The highresolution mineralogical data are co-registered and resampled to the new high-resolution hyperspectral data following our strategy in [13]. Another advantage of this method is that, if SEM-MLA data are available, once these have been co-registered, the number of training samples also increases in comparison to the method proposed in [13] because of the enhanced spatial sampling, which is crucial for accurate machine learning estimations.

As expected, once the hyperspectral data have been enhanced, the amount of data considerably increases (i.e., 7.3 MB to 1.6 GB for one drill-core interval of ca. $30 \mathrm{~cm}$ ). While the technique can be applied to large datasets, we present the hyperspectral data of three relevant drill-core samples of about $30 \mathrm{~cm}$ to showcase the approach. The hyperspectral data cover the VNIR-SWIR region of the electromagnetic spectrum with 450 bands.

The manuscript is organized into six sections: Section 2 presents the proposed methodology for resolution enhancement for mineral mapping. Section 3 describes the datasets used in this study, the parameter settings, and the application. Section 4 describes the experimental results achieved. Section 5 describes a critical assessment of this study, and finally, the conclusions are presented in Section 6. 


\section{Methods: Resolution Enhancement for Mineral Mapping}

Our proposed methodology to improve spatial details when mapping minerals in complex natural samples using high-resolution hyperspectral data is schematically illustrated in Figure 1. We generate a high-spatial and spectral resolution hyperspectral dataset by fusing high-spatial resolution RGB with hyperspectral drill-core data using a subspacebased resolution enhancement algorithm (i.e., coupled non-negative matrix factorization (CNMF) [22]). On one hand, the enhanced hyperspectral data can already be used to generate high-spatial resolution mineral maps based on detailed spectral parameters, using, for example, a spectral library. On the other hand, we can obtain high-resolution classified maps of the drill-core samples. For this purpose, we prepare the training data by co-registering and resampling high-resolution mineralogical data with the new hyperspectral data. We then use a supervised machine learning algorithm (i.e., Canonical Correlation Forest $(\mathrm{CCF})$ ) to train a model and further classify the enhanced drill-core hyperspectral data.

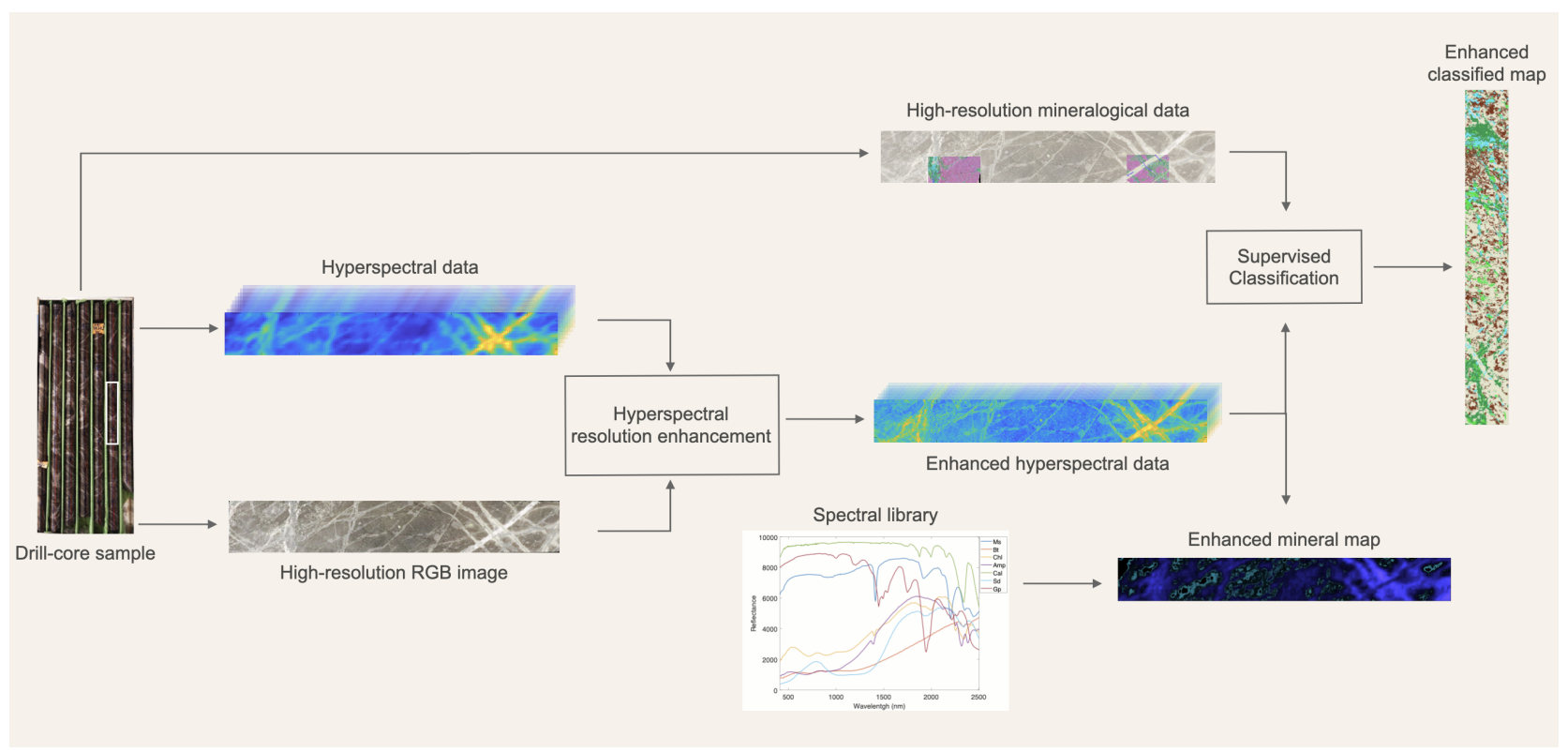

Figure 1. Flowchart of the proposed methodology to map minerals at a high-spatial resolution using hyperspectral data.

\subsection{Hyperspectral Resolution Enhancement}

The spatial sampling of hyperspectral sensors often results in a limited resolution of details and patterns in mineral maps. SEM-MLA analysis provides a mineral map at highspatial resolution (i.e., GSD of about $3 \mu \mathrm{m}$ ) but to an extremely limited spatial extent (e.g., covering a region of about $39 \times 29 \mathrm{~mm}$ ). To improve spatial sampling of the hyperspectral data and to facilitate a more meaningful use of the SEM-MLA analysis as reference data, we enhance the resolution of the hyperspectral data by means of a high-spatial resolution RGB image of the same sample.

We perform the enhancement with the Coupled Non-negative Matrix Factorization algorithm (CNMF [22]). We chose this algorithm for its robust performance as a benchmark method, as shown in the literature, and for its ability to preserve the detailed spectral information in the enhanced hyperspectral data [22,25], which represents a key factor when mapping minerals. Moreover, CNMF has been shown to perform better than a variety of resolution enhancement methods, e.g., [21,26]. CNMF is a resolution enhancement algorithm belonging to the family of subspace methods. It was originally proposed to fuse hyperspectral, $\mathbf{X} \in \mathbb{R}^{\lambda_{h} \times L_{h}}$, and multispectral data, $\mathbf{Y} \in \mathbb{R}^{\lambda_{m} \times L_{m}}$, based on a recursive non-negative matrix factorization (NMF) unmixing. $\lambda_{h}$ and $\lambda_{m}$ represent the number of spectral bands in the hyperspectral and multispectral data, whereas $L_{h}$ and $L_{m}$ denote the number of pixels in both datasets. The fusion of hyperspectral and multispectral data, 
which in this study correspond to a three-spectral-channel RGB image, based on unmixing is achieved by estimating high-spectral resolution endmember spectra $(\mathbf{W})$ and high-spatial resolution abundances maps $(\mathbf{H})$ from the two datasets [22]. Spectral unmixing of the multispectral image itself cannot be achieved accurately due to the broad spectral response at limited wavelengths. However, this unmixing is helpful to determine the detailed spatial distribution of abundances at fine resolutions once the spectral signatures and the fractional abundances are obtained at a lower spatial resolution by spectral unmixing of the hyperspectral data [25].

CNMF starts by unmixing the low-spatial resolution hyperspectral data. For the unmixing, a linear model is adopted due to its physical effectiveness and mathematical simplicity. CNMF alternately unmixes $\mathbf{X}$ and $\mathbf{Y}$ using non-negative matrix factorization (NMF) to estimate $\mathbf{W}$ and $\mathbf{H}$, and for this, the following constraints are considered: the endmembers and abundances are assumed to be non-negative $(\mathbf{W} \geq 0$ and $\mathbf{H} \geq 0)$ and the abundances are also constrained to sum-to-one $\left(\sum_{j=1}^{D} h_{j k}=1\left(k=1,2, \ldots, L_{m}\right)\right.$, where $D$ is the number of endmembers). Moreover, NMF does not assume the presence of pure pixels. For unmixing with NMF, the CNMF algorithm uses multiplicative update rules, which are as follows:

$$
\begin{aligned}
& \mathbf{W} \leftarrow \mathbf{W} \cdot *\left(\mathbf{X H}_{h}^{T}\right) \cdot /\left(\mathbf{W H}_{h} \mathbf{H}_{h}^{T}\right) \\
& \mathbf{H}_{h} \leftarrow \mathbf{H}_{h} \cdot *\left(\mathbf{W}^{T} \mathbf{X}\right) \cdot /\left(\mathbf{W}^{T} \mathbf{W} \mathbf{H}_{h}\right) \\
& \mathbf{W}_{m} \leftarrow \mathbf{W}_{m} \cdot *\left(\mathbf{Y} \mathbf{H}^{T}\right) \cdot /\left(\mathbf{W}_{m} \mathbf{H} \mathbf{H}^{T}\right) \\
& \mathbf{H} \leftarrow \mathbf{H} \cdot *\left(\mathbf{W}_{m}^{T} \mathbf{Y}\right) \cdot /\left(\mathbf{W}_{m}^{T} \mathbf{W}_{m} \mathbf{H}\right)
\end{aligned}
$$

where $(\cdot)^{T}$ denotes the transposed matrix and where $* *$ and $\cdot /$ denote element wise multiplication and division, respectively.

In the initial phase, the endmember matrix is calculated by the vertex component analysis algorithm (VCA). The abundance matrix from the hyperspectral data (Equation (2)) is set as a constant value $1 / D$ and is updated until convergence with the endmembers fixed. For the optimization of $\mathbf{W}$ and $\mathbf{H}_{h}$, they are alternately updated using Equations (1) and (2) until the next convergence. In the subsequent NMF unmixing for $\mathbf{X}$, the initialization phase for the abundances is performed considering the point spread function. The endmembers (Equation (1)) are updated until convergence with abundances from the hyperspectral data, which is important to inherit the reliable information of abundance maps obtained from multispectral data [22]. In the optimization phase, both $\mathbf{W}_{m}$ and $\mathbf{H}$ are alternately updated using Equations (3) and (4) until the next convergence.

The point spread function, together with the spectral response function, are sensordependent characteristics that are related to the input hyperspectral and multispectral data by building sensor observation models into the initialization of the multispectral signatures of the endmembers and the low-spatial resolution abundance maps [21]. The relative sensor characteristics can be estimated from the data sources when they are not available as prior knowledge. The spectral response function is used to initialize the endmember matrix derived from the NMF unmixing of the high-spatial resolution RGB image. The abundances from the multispectral data are also initialized in this step as the constant value $1 / D$ and are updated until convergence with the endmembers from the multispectral data. Through this process, reliable information about the endmember spectra obtained from the hyperspectral data is inherited [22]. The two NMF unmixing steps are repeated alternately until convergence, and the final high-spatial resolution hyperspectral data are obtained by deriving the product of both $\mathbf{W}$ and $\mathbf{H}$. For a more detailed definition of the CNMF algorithm, we refer the readers to [22].

\subsection{Mineral Mapping}

We generate initial mineral maps from the enhanced hyperspectral data using a wellestablished and robust minimum wavelength mapper. We use the version available in the Hylite toolbox for spectral analysis [27]. The minimum wavelength mapper combines the 
position and depth information of the deepest absorption feature to provide an overview of its presence and distribution $[10,28]$. Changes in the wavelength position are observed in color variations, and hue variations represent changes in the strength of the absorption feature. We perform the mapping on continuum-removed data and evaluate the position and depth of the deepest absorption feature present in the data. We perform the mapping between 2130 and $2370 \mathrm{~nm}$ since, based on a spectral library [29], this wavelength range encloses the diagnostic absorption features of minerals such as white micas, chlorites, carbonates, and kaolinites, amongst others.

\subsection{Supervised Classification}

After the hyperspectral data were enhanced, we co-registered and resampled the SEM-MLA data to match the enhanced hyperspectral data and to generate the training or reference data to perform the supervised mapping. For this, we followed a modified approach from the one presented by [13]. We opted to resample the SEM-MLA data by selecting the most dominant minerals per hyperspectral pixel instead of generating class labels with their associated mineral abundances. This is because the spatial sampling of the high-spatial resolution hyperspectral image is now more related to sampling the original high-resolution mineralogical data used in this study. A threshold needs to be set to guarantee the presence of representative minerals (i.e., minerals with a minimum abundance in the entire region covered by the SEM-MLA data) for resampling. We consider all of the minerals identified in the SEM analysis. However, minerals that do not have a diagnostic response in the spectral range used for the hyperspectral data (VNIR-SWIR) have been set as one class called Others in the final labelling of the resampled maps. These non-diagnostic minerals are, for example, quartz, plagioclase, and pyrite.

The last step of the proposed methodology concerns upscaling of the high-resolution mineralogical data from the SEM-MLA to the drill-core samples and is performed by training a machine learning model. To train the model, the hyperspectral features are given as input together with the training samples to a supervised classifier. In this work, we suggest using a novel decision tree ensemble method, i.e., Canonical Correlation Forest (CCF) [30], since it is known that ensemble classifiers can provide good classification results where there is no balance between dimensionality and the number of available training samples. Moreover, the combination of decision trees in ensemble classifiers is expected to provide more accurate and robust results than individual decision tree classifiers.

CCF applies canonical correlation analysis to capture the relationship between the class labels and features before the leaf split is performed in each decision tree [31]. It applies the correlation analysis in the construction of trees to find feature projections that provide the maximum correlation between the features and the class labels. The best split at a particular node in this projected space is selected using an exhaustive search in the space of the projected features. CCF is constructed by several individual canonical correlation trees. The individual trees are binary decision trees with hyperplane splits based on local canonical correlation coefficients calculated during the training [30]. Majority voting is used to combine the predictions of the individual canonical correlation trees.

\section{Case Study}

We showcase the performance of our proposed methodology on three drill-core samples from the Bolcana copper-gold mineralized system located in the Brad-Sacaramb metallogenic district within the Golden Quadrilateral in South Apuseni Mountains, Romania. This is a porphyry-type $\mathrm{Cu}-\mathrm{Au}$ ore deposit with associated epithermal veins [32]. Hyperspectral data were acquired over the surface of these drill-cores, and small portions of them were subjected to the SEM-MLA analysis (see Figure 2). In Sample 1, the matrix is predominantly composed of white mica, and a large pyrite-gypsum vein is presented. Sample 2 and Sample 3 are composed of mainly feldspars with disseminated chlorite and biotite in the matrix at different proportions. Sample 2 has minor chlorite, whereas Sample 3 has less biotite. Sample 2 presents fine pyrite and quartz veins, whereas Sample 3 presents 
pyrite-gypsum veins and quartz veins. From these minerals only white mica, biotite, chlorite, and gypsum have diagnostic responses in the VNIR-SWIR wavelength range.

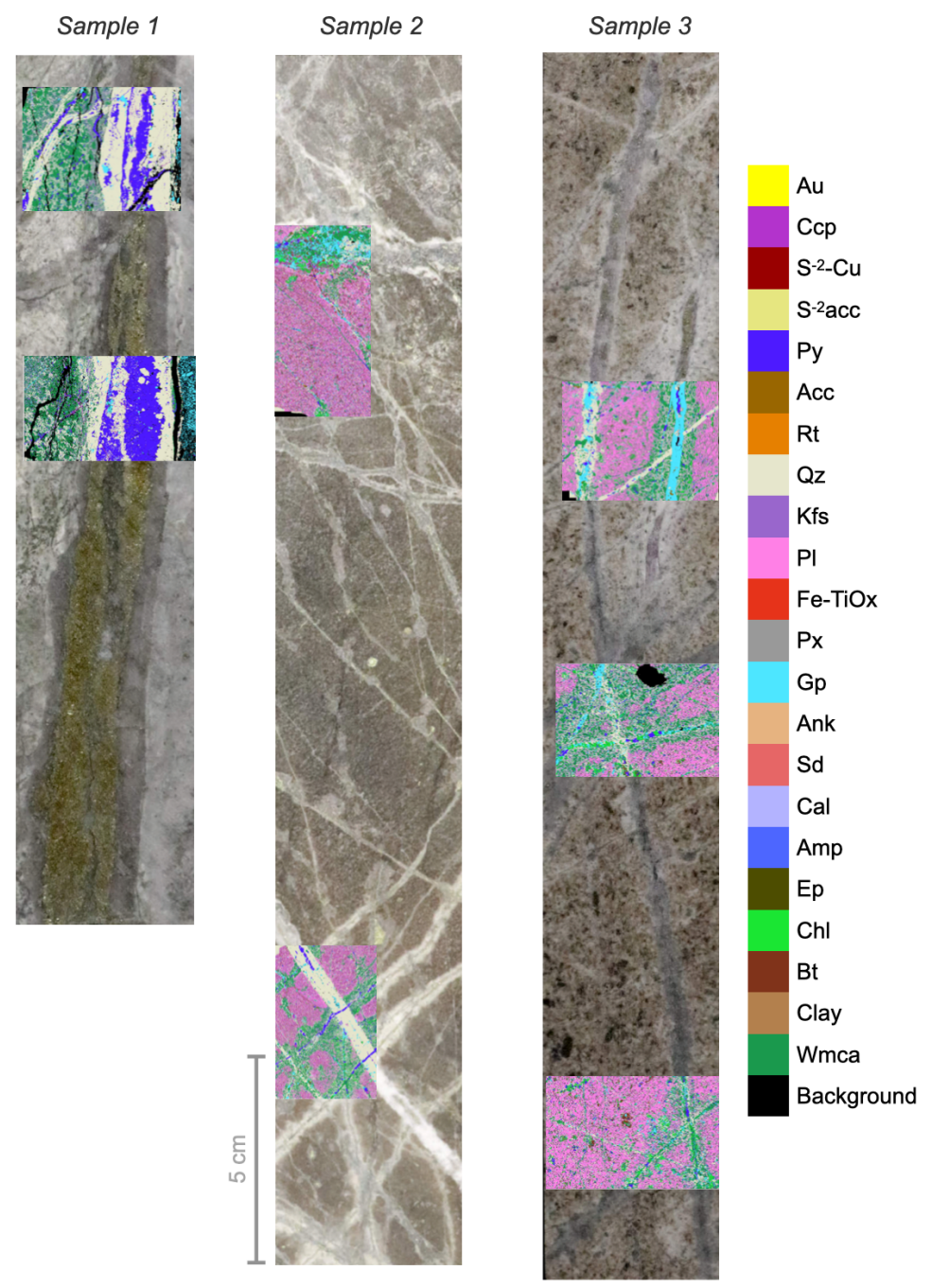

Figure 2. RGB images of the drill-core samples used in this study with the overlaid SEM-MLA images. The pixel size in the original SEM-MLA images is about $3 \mu \mathrm{m}$. (Wmca—white mica, Bt—biotite, Chl—chlorite, Ep-epidote, Amp-amphibole, Cal—calcite, Sd—siderite, Ank—ankerite, Gp—gypsum, Px—pyroxenes, Fe-TiOx—Fe-Ti oxides, Pl—plagioclase, Kfs— K-feldspar, Qz-quartz, Rt—rutile, Acc—accessory minerals, Py—pyrite, $\mathrm{S}^{-2}$ acc—sulphides accessory, $\mathrm{S}^{-2}$-Cu-copper sulphides, Ccp-chalcopyrite, and Au-gold).

\subsection{Data Acquisition}

For the acquisition of data, a high-resolution RGB and a hyperspectral camera were mounted onto the SisuRock scanner manufactured by SPECIM (see Figure 3a). The scanner is an automatic imaging workstation that carries the samples placed on a moving table under the field-of-view of the cameras. We used a Canon EOS 750D for high-resolution RGB data. This is a 24.2 megapixel digital SLR camera that produces high-resolution RGB images of about $0.1 \mathrm{~mm}$ ground sampling distance (GSD).

For the hyperspectral data, we used the SPECIM FENIX camera. This is a push-broom instrument that contains two sensors to cover the VNIR-SWIR regions of the electromagnetic spectrum, 380-970 nm and 970-2500 nm, respectively. The spectral resolution of the VNIR sensor is about $3.5 \mathrm{~nm}$ and that of the SWIR sensor is about $12 \mathrm{~nm}$. The spectral binning was set to 4 for the VNIR and to 1 for the SWIR. The scanning speed was set to $25.06 \mathrm{~mm} / \mathrm{s}$, and the integration times were $15 \mathrm{~ms}$ and $4 \mathrm{~ms}$ for the VNIR and SWIR, 
respectively. The FENIX camera provides a co-register hyperspectral data cube of the VNIR-SWIR data with a total of 450 bands and a GSD of around $1.5 \mathrm{~mm}$.

The radiance values of the hyperspectral data were automatically converted to reflectance values based on an empirical line correction with a dedicated plugin in the acquisition software provided by SPECIM. We also applied geometric corrections using the toolbox presented in [33] to account for the sensor-specific optical distortions known as fish-eye and slit-bending effects. We removed the hyperspectral data from 380 to $538 \mathrm{~nm}$ and from 2486 to $2500 \mathrm{~nm}$ to avoid bands with low signal-to-noise ratio. Hence, the hyperspectral data we used for this work have a total of 400 bands.

The high-resolution mineralogical data were acquired using SEM-MLA with an FEI Quanta $650 \mathrm{~F}$ field emission SEM instrument, equipped with two Bruker Quantax X-Flash 5030 energy dispersive X-ray detectors and the MLA 3.1.4 software (see Figure 3b). The acquisition was performed in GXMAP mode over carbon-coated polished thin sections of about $30 \mu \mathrm{m}$ thickness from the drill-core samples. The GSD of the MLA images is about $3 \mu \mathrm{m}$. More details on the measurement mode are available in [34,35].

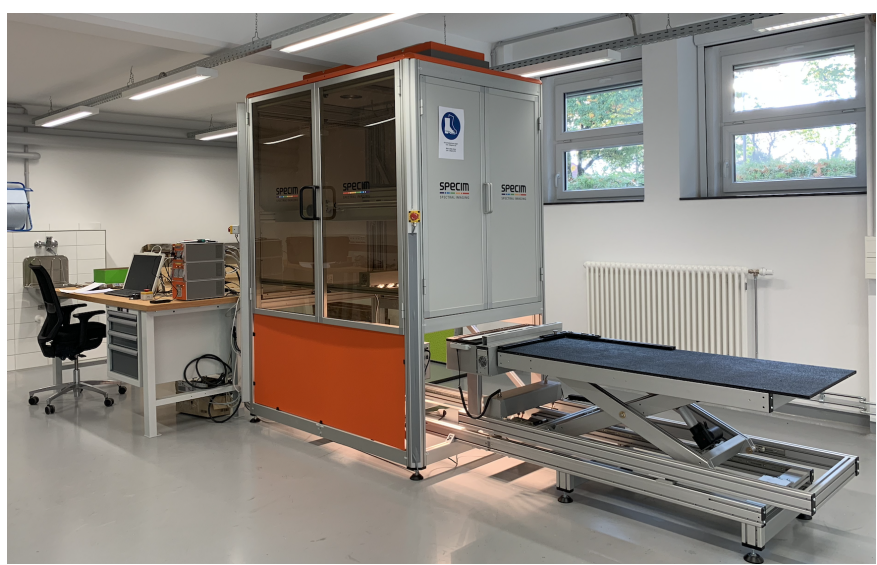

(a) SisuRock drill-core scanner equipped with an AisaFenix VNIR-SWIR hyperspectral sensor.

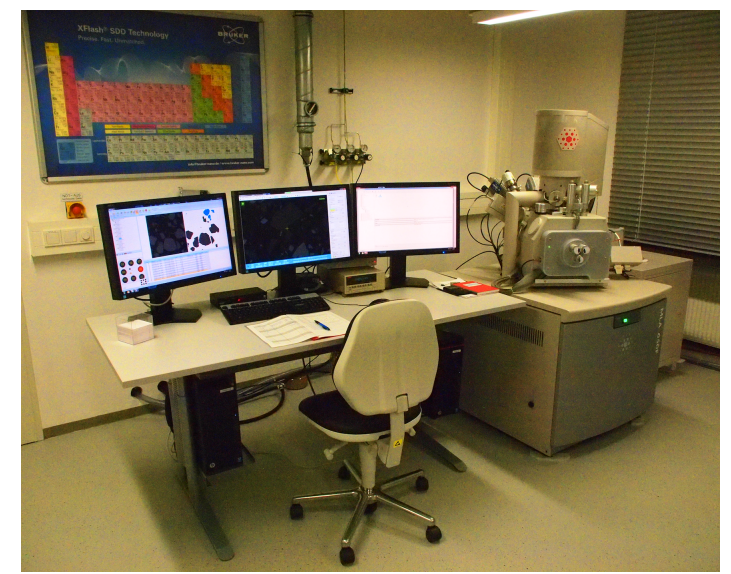

(b) Scanning Electron Microscope (SEM)Mineral Liberation Analysis (MLA).

Figure 3. Drill-core scanner and SEM-MLA instruments available at the spectroscopy and geometallurgy laboratories, respectively, at the Helmholtz Institute Freiberg for Resource Technology.

\subsection{Resolution Enhancement Application}

For the resolution enhancement of hyperspectral data using the CNMF algorithm, the number of endmembers can be set manually or can be determined automatically using virtual dimensionality (VD) [36]. In this case, we opted for automatic estimation of the endmembers and set the false alarm rate of the VD to 0.05 to guarantee the detection of weak signal sources with small energies [36]. The ratio between spatial sampling of the hyperspectral and RGB data is the enhancement factor (EF). The three samples used in this study have EFs ranging between 15 and 17. These factors are reasonable considering that one spectrum from the original hyperspectral dataset is used to determine the spectral content of only 225-289 pixels. With these EFs the spatial sampling of the hyperspectral data is decreased from about $1.5 \mathrm{~mm}$ to $0.1 \mathrm{~mm}$. Mineral sizes vary from microns to millimeters in the rock samples considered. That implies that one pixel from the enhanced dataset is more likely to contain a single mineral (pure pixels).

The threshold we used to generate the training set from the resampled SEM-MLA data corresponds to around $2 \%$ of the total pixel area covered by the SEM-MLA. We chose this value by testing different options and by assessing for a meaningful number of minerals considering the GSD of the hyperspectral data. We also performed the mapping with the GSD of the original hyperspectral data to be able to evaluate the mapping improvement at 
the enhanced sampling. In this case, we set the same value for the threshold in resampling the SEM-MLA data.

We selected the number of training samples at $80 \%$ of the smallest class to avoid unbalanced classes. This step is necessary because, for example, the class Others is highly abundant and mainly consists of mixtures of minerals without a diagnostic response in the VNIR-SWIR. The performance evaluation of the classifier is not a relevant point in this study but the actual mapping of enhanced sampling is. Additionally, one should be aware that using traditional metrics to evaluate an improvement in mapping for enhanced sampling is not completely appropriate since mapping at the original and enhanced GSD are two different classifications based on two different training sets. However, for the sake of transparency, we calculate the accuracies of the CCF algorithm when mapping at the original and enhanced resolutions. The number of samples in the validation set is chosen as $20 \%$ of the smallest class. We used the values suggested in the literature to tune the CCF algorithm [30,31] and used 500 trees for the classification because this number of trees has been shown to produce stable results when mapping minerals [13].

The resolution enhancement in this study derives datasets in which a more precise delineation of elements is observed due to the finer spatial sampling of the hyperspectral data. For example, in the enhanced image of the area within the red square in Figure 4, the total number of pixels corresponds to $345 \times 345$ whereas the original dataset for this area has $23 \times 23$ pixels. This increment depicts the elongated and linear features better and not only the thicker ones as in the original data. The relevance of this enhancement lies in the fact that these linear features correspond to veins that are highly important for geologists looking for indicators of ore mineralizations.
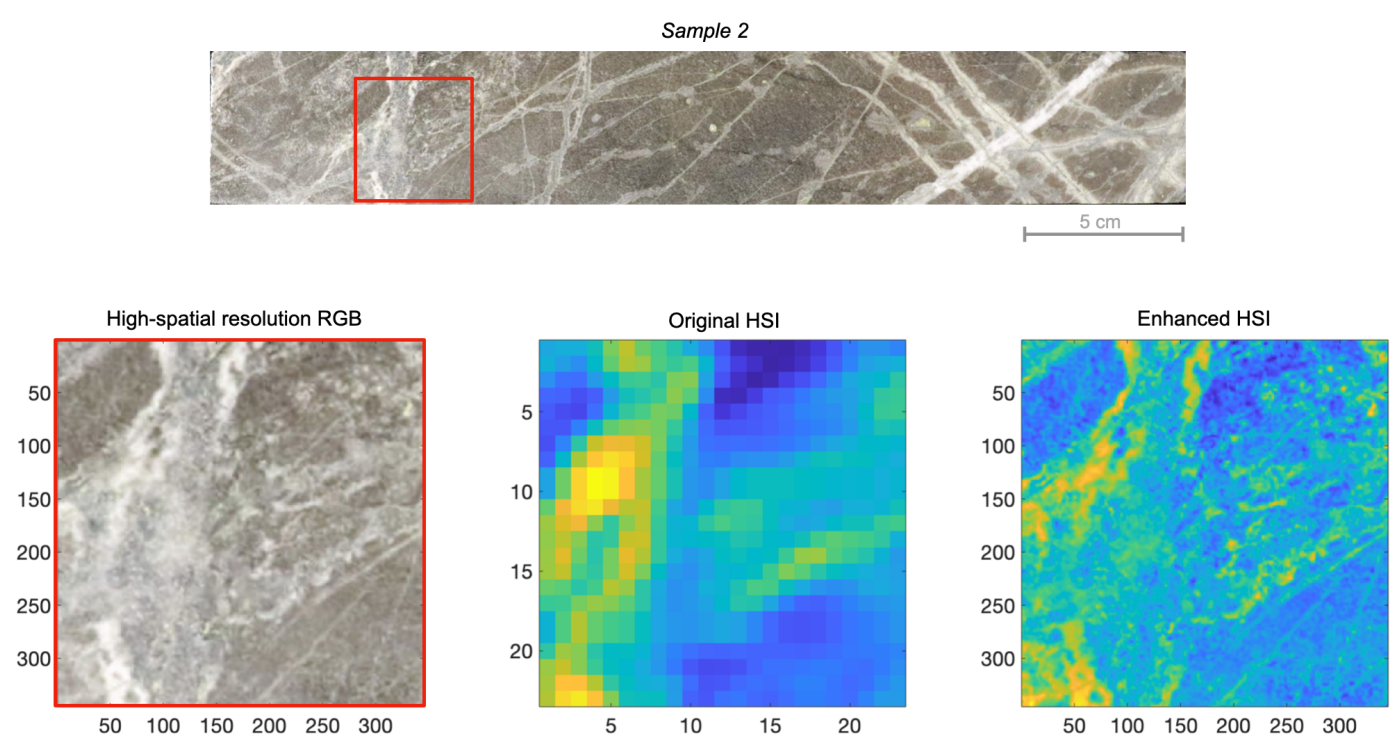

Figure 4. Resolution enhancement example: RGB image of Sample 2 with red square showing an specific region where the enhancement is illustrated. Bottom row shows the RGB image of the specific region, band $889 \mathrm{~nm}$ of the original hyperspectral (HSI) data (1.5 mm ground sampling distance (GSD)) and the same band of the enhanced hyperspectral data (0.1 mm GSD).

\subsection{Evaluation of the Resolution Enhancement}

We performed a sensitivity analysis to evaluate whether the spectral fidelity is preserved in the resolution enhancement. We used the spectral angle mapper (SAM) [37], which is a widely used quality measure for quantitative fusion assessment. SAM is a common measure in the field of resolution enhancement for determining the spectral preservation at each pixel $[21,25]$. It determines the spectral similarity between two spectra, in this case, between the enhanced and reference spectra (original dataset), by calculating the angle between their vectors in the spectral space. For the enhancement, we used not 
only the original RGB data but also six extra datasets obtained by down-sampling the original RGB image to also assess the impact of the EF in the spectra. The EF of these datasets ranges from 3 to 13 , and the total number of pixels varies from $69 \times 69$ to $345 \times 345$. We considered an average of 200 pixels for each dataset when calculating the spectral similarity to produce more sound results. The sensitivity analysis shows stable results with average values around $2.2 \mathrm{rad}$ for all of the datasets generated with the different $\mathrm{EF}$. The dataset with the closest sampling to the original hyperspectral data (EF of 3 ) and the one obtained with the original RGB data (EF 15) are the ones presenting slightly lower values (see the plot at the bottom right of Figure 5). The sensitivity analysis shows that the reconstruction step captures the main absorption features and general shape of the spectra in the enhancement process. This can be also observed in the plot at the bottom left of Figure 5 where the only apparent difference is visible in the reflectance intensity for all of the enhanced data. These spectra correspond to the center pixels marked with the red dots in the hyperspectral images (HSI) and MLA image overlaid on the RGB of the section of interest from Sample 2 shown at the top of the figure. When using hyperspectral data to map minerals, the absorption features are crucial for proper identification of the present minerals. Based on the wavelength location of the main absorption features of the spectra shown in Figure 5, these show a dominant presence of biotite.

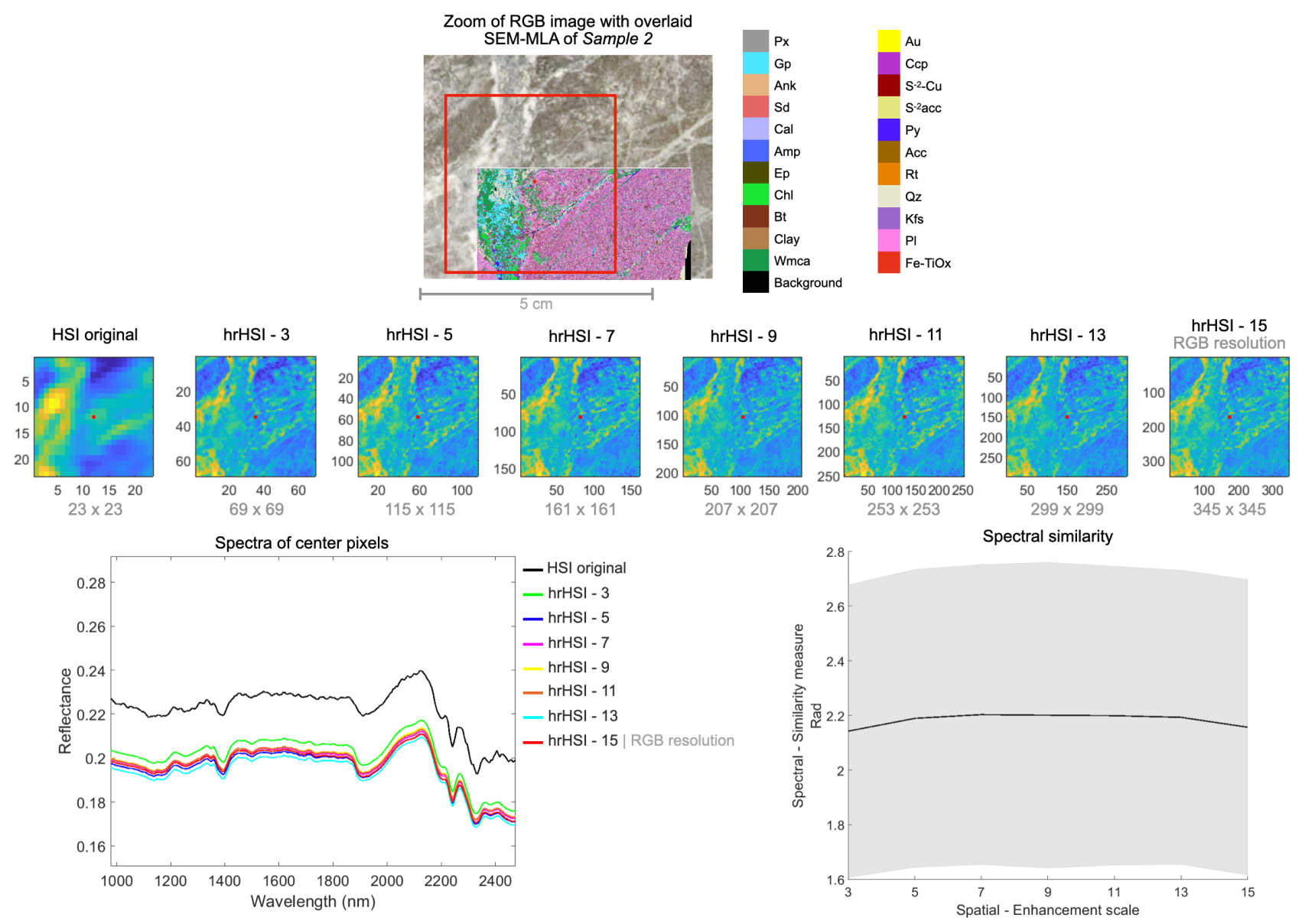

Figure 5. Sensitivity analysis for the spectral integrity by means of SAM measurement for a small section of Sample 2. Top image shows an RGB image of the small section, and the red square shows the area where the analysis was performed. Below are images of band $889 \mathrm{~nm}$ of the hyperspectral (HSI) data enhanced at different factors with an overlaid red dot pointing toward the center pixel. At the bottom, from left to right, is the spectra of the center pixels and the plot with the SAM results. (Wmca—white mica, Bt—biotite, Chl—chlorite, Ep—epidote, Amp—amphibole, Cal—calcite, Sd—siderite, Ank—ankerite, Gp—gypsum, Px—pyroxenes, Fe-TiOx—Fe-Ti oxides, Pl—plagioclase, Kfs—K-feldspar, Qz-quartz, Rt— rutile, Acc-accessory minerals, Py-pyrite, $\mathrm{S}^{-2}$ acc—sulphides accessory, $\mathrm{S}^{-2}-\mathrm{Cu}$-copper sulphides, Ccp-chalcopyrite, and $\mathrm{Au}-$ gold). 


\section{Results \\ 4.1. Mineral Mapping}

Initial high-spatial resolution mineral maps obtained by the minimum wavelength mapper serve to evaluate the spectral parameters, wavelength position, and depth of the main absorption features, which relate to the minerals present in the samples (see Figure 6). The main color present in the matrix of Sample 2 and Sample 3 is representative of chlorite and biotite rich in magnesium. The intense blue in the veins is representative of mica richer in magnesium or iron (more phengitic composition), while the magenta color in the middle and upper part of the veins in Sample 3 and Sample 1 corresponds to the mica of a paragonitic composition.

Minimum wavelength maps are also useful for assessing the performance of the resolution enhancement and whether the spectra have been properly preserved by visually comparing the high-spatial resolution mineral maps with the maps obtained with the original hyperspectral data. In general, the enhanced mineral maps show a striking improvement in the delineation of the structures such as veins. For example, in Figure 6, the first vertical vein at the bottom left part of Sample 3 is not mapped in the enhanced HSI as sharp as it is in the original HSI. This vein is visible in the SEM-MLA maps in Figure 7 at the bottom, and it is mainly composed of quartz $(\mathrm{Qz})$, which does not have diagnostic absorption features in the VNIR-SWIR wavelength range and, therefore, should not be highlighted in the minimum wavelength maps. Another example is the sharp vertical vein at the bottom right of Sample 3 (see Figure 6), which in the SEM-MLA maps in Figure 7 corresponds to the vein at the top. This vein is composed of a mixture of quartz with white mica (Wmca) and less chlorite (Chl). The presence of white mica is what sharpens the vein in the enhanced minimum wavelength maps.

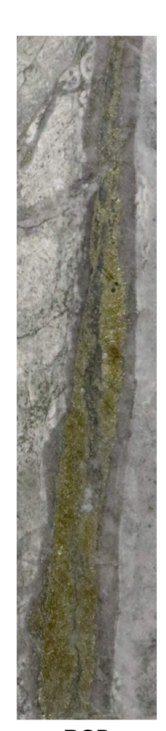

RGB
Sample 1

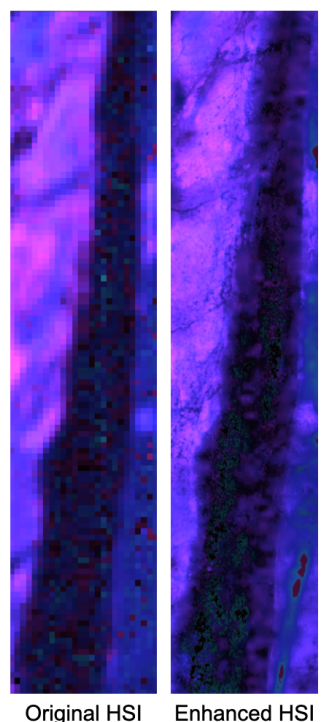

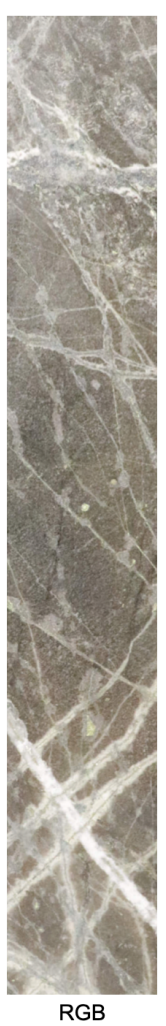

Sample 2

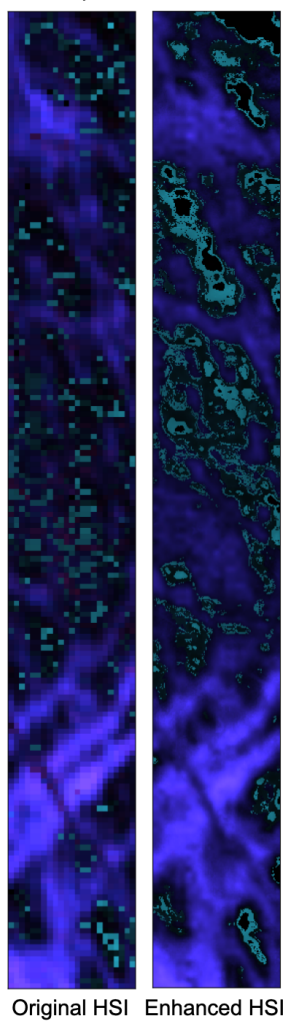

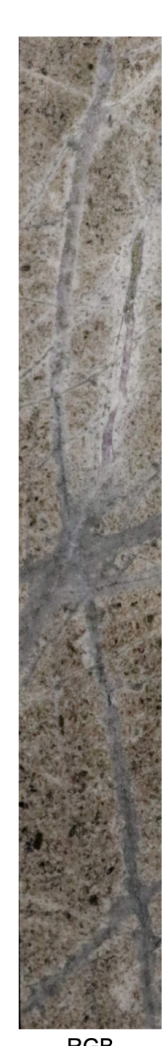

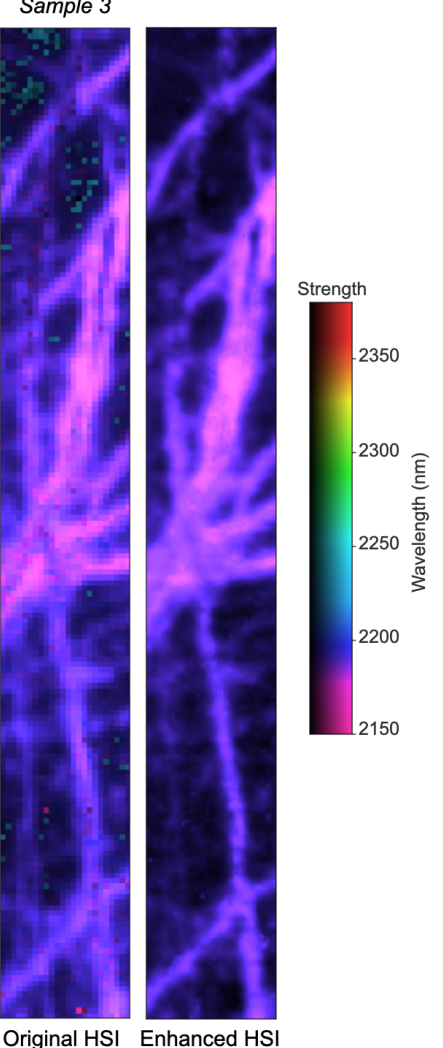

Figure 6. Minimum wavelength maps performed over the range between 2130 and $2370 \mathrm{~nm}$ for both the original and enhanced hyperspectral images (HSI). A stretch for visualization was performed between 2150 and $2370 \mathrm{~nm}$. 


\subsection{Supervised Classification}

The enhanced hyperspectral data allows for more meaningful use of SEM-MLA data to train a supervised machine learning model for the mineral mapping of drill-cores because of the smaller GSD. With the smaller GSD achieved in the enhanced data, the difference in the spatial sampling of the SEM-MLA and hyperspectral data is minimized, and therefore, smaller objects such as mineral grains are depicted in the matrix. With the SEM-MLA resampled to the enhanced GSD, not only are higher details mapped but also more minerals can be preserved from the original SEM-MLA. For example, a region could only contain one mineral at a $1.5 \mathrm{~mm}$ GSD, but when increasing the resolution to $0.1 \mathrm{~mm}$ GSD, the region shows more than just one mineral. To illustrate the improvement in the use of the SEM-MLA data, Figure 7 shows one of the SEM-MLA images obtained from Sample 3 and the resampled versions for both resolutions: the original hyperspectral and enhanced data. In general, the matrix of the sample at the original resolution shows only plagioclase, which is a mineral without diagnostic absorption features in the VNIR-SWIR. However, the image resampled to the resolution of the enhanced hyperspectral data shows that, in the matrix, there are in fact grains of other minerals that have a diagnostic response in the wavelength range used(see also the zoom map at the bottom right of the figure). For example, the grain within the red sphere in the zoom is mapped as biotite. The average spectra of the area within the red sphere show the characteristic absorption features of biotite, however, not for pure biotite (see the spectra at the bottom left of the figure).
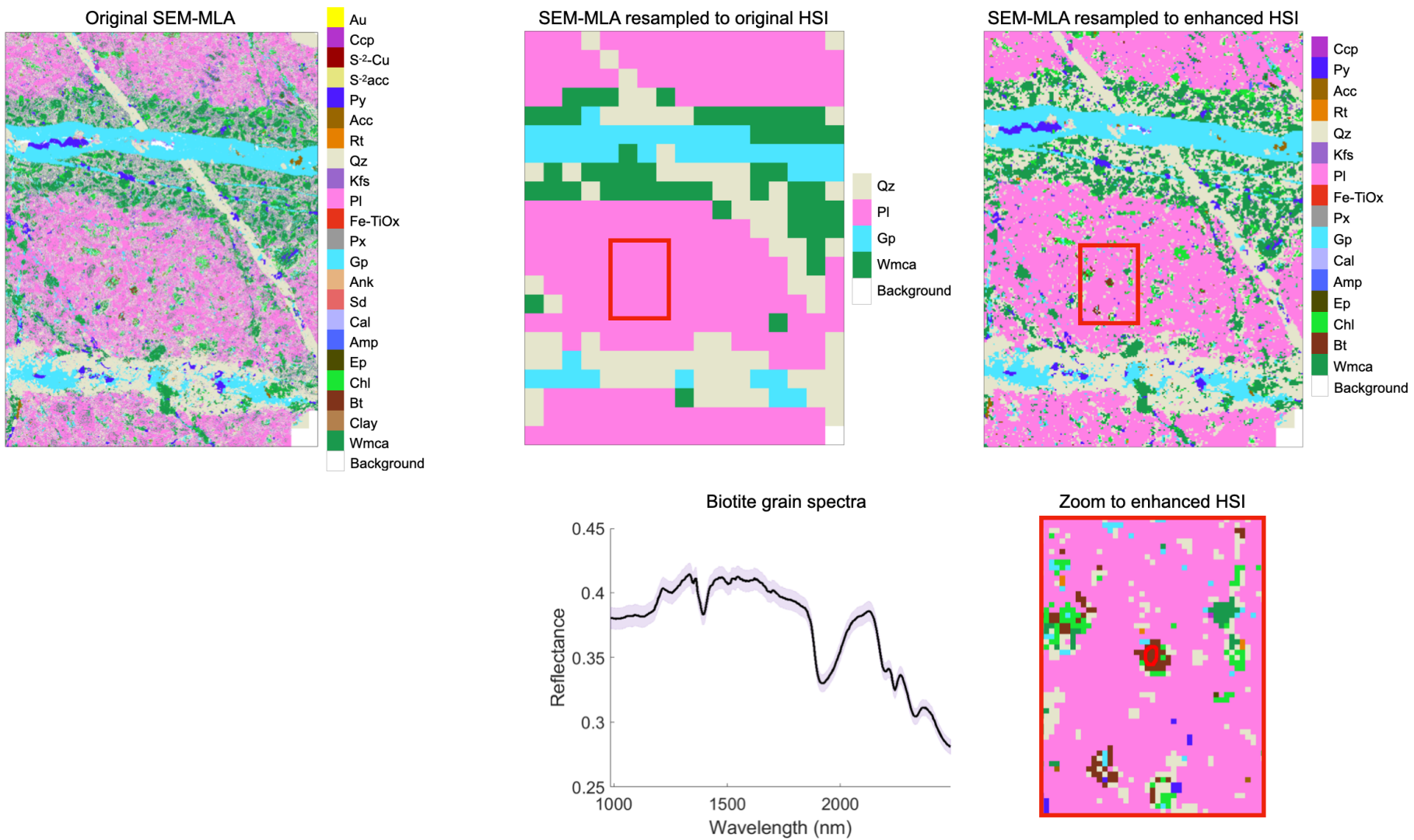

Figure 7. SEM-MLA performed over a small section of Sample 3 (top section in Sample 3 in Figure 2) at the original resolution ( $3 \mu \mathrm{m}$ GSD), resampled to the original resolution of the hyperspectral (HSI) data (1.5 mm GSD), and resampled to the enhanced HSI resolution (0.1 mm GSD). Below is a zoom of the red squared over the enhanced HSI map and the spectra of the biotite (Bt) grain pointed with the red sphere. (Wmca-white mica, Bt—biotite, Chl—chlorite, Ep-epidote, Amp—amphibole, Cal—calcite, Sd—siderite, Ank—ankerite, Gp-gypsum, Px—pyroxenes, $\mathrm{Fe}-\mathrm{TiOx}$ - $\mathrm{Fe}-\mathrm{Ti}$ oxides, $\mathrm{Pl}$ plagioclase, Kfs-K-feldspar, Qz-quartz, Rt—rutile, Acc—accessory minerals, Py—pyrite, $\mathrm{S}^{-2}$ acc—sulphides accessory, $\mathrm{S}^{-2}-\mathrm{Cu}$-copper sulphides, $\mathrm{Ccp}$-chalcopyrite, and $\left.\mathrm{Au}-\mathrm{gold}\right)$. 
The training data were obtained after the original SEM-MLA images were co-registered and resampled to the resolution of the enhanced hyperspectral data (from $3 \mu \mathrm{m}$ GSD to $0.1 \mathrm{~mm}$ GSD) (see maps on the right side of Figure 8). Following the same approach, we also performed resampling of the high-resolution mineralogical maps to the original resolution of the hyperspectral data (from $3 \mu \mathrm{m}$ GSD to $1.5 \mathrm{~mm}$ GSD) to compare the high-spatial resolution maps (see left side of Figure 8). SEM-MLA reference maps at enhanced resolution show more detailed patterns as more linear and circular features, which correspond to veins and mineral grains, are delineated. Additionally, the model mineralogy provided by SEM-MLA is explored to its fullest extent. This observation is evident in the matrix of Sample 2 and Sample 3, where mineralogical changes are observed. At the original resolution, the matrix contains mainly the class Others, and at the enhanced resolution, it is composed of biotite $(B t)$ and chlorite (Chl) besides the class Others. These changes are also evident in the veins of Sample 1 and Sample 3. To illustrate this, the top SEM-MLA in Sample 1 shows, in contrast to the map at the original resolution, that the vein is composed of white mica and not only of the class Others.

SEM-MLA based training data at original HSI resolution
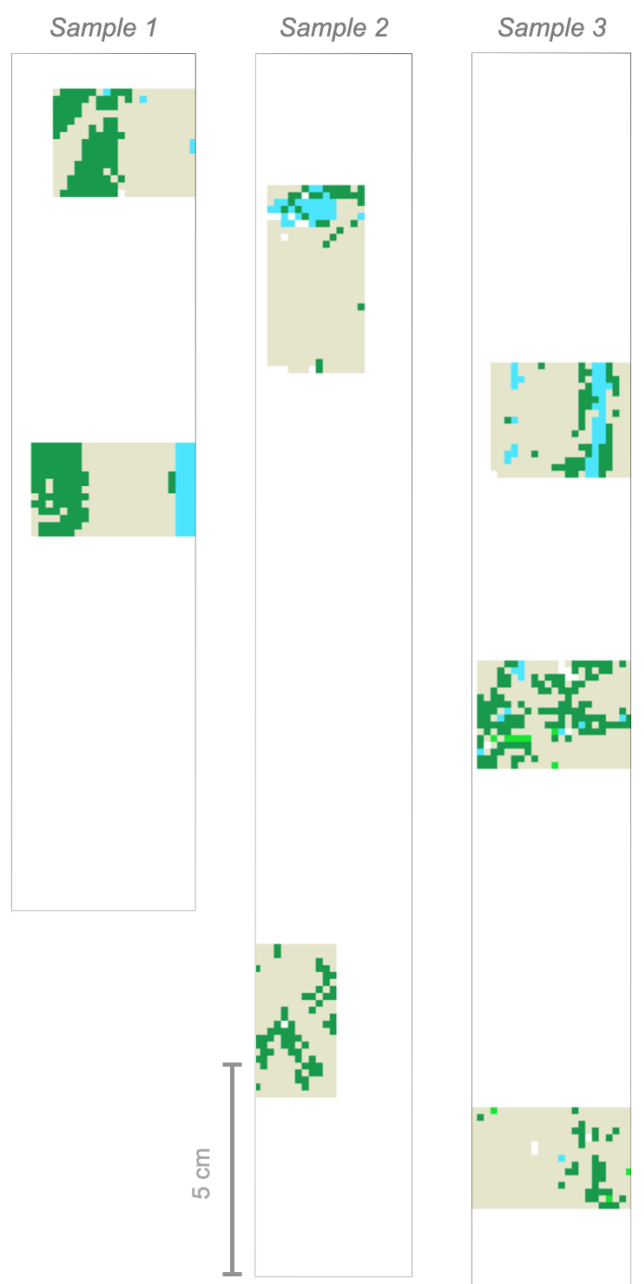

SEM-MLA based training data at enhanced HSI resolution

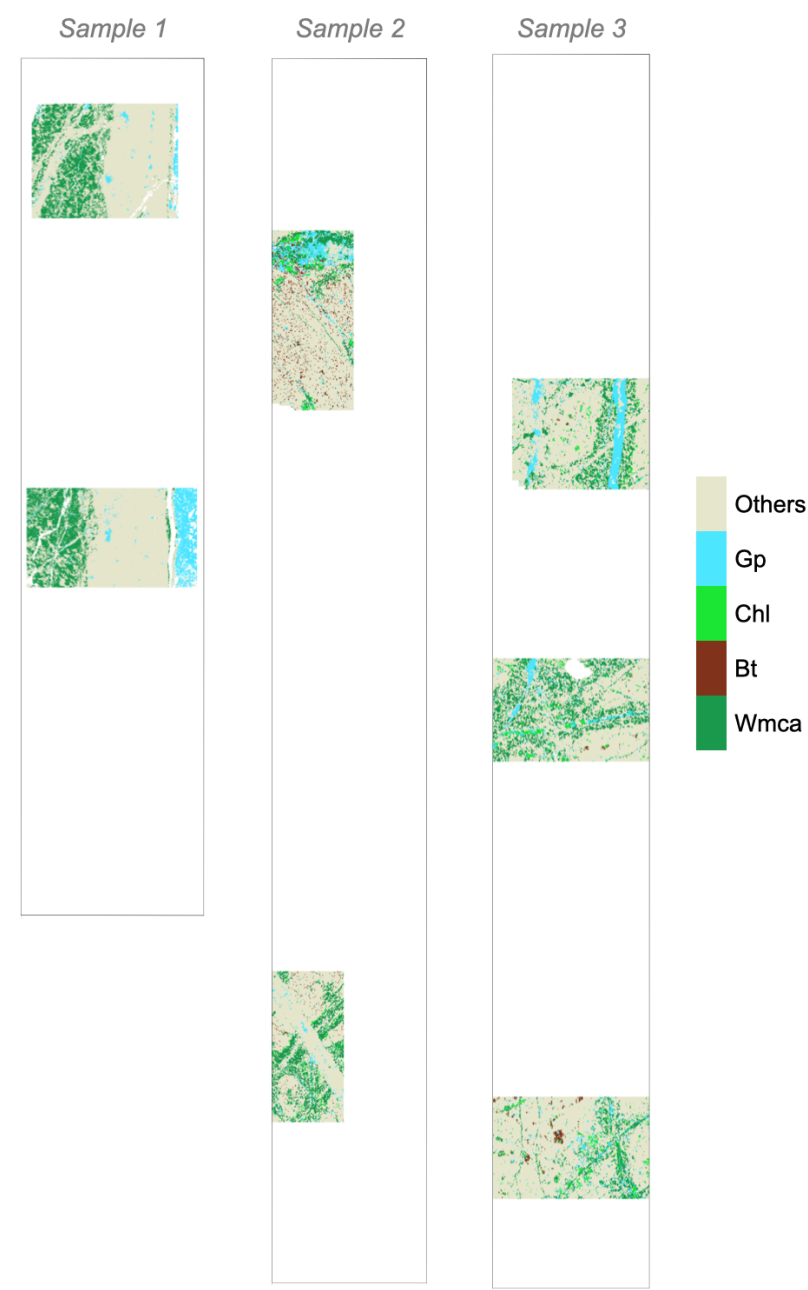

Figure 8. Co-registered scanning electron microscopy-mineral liberation analysis (SEM-MLA) images and resampled to the original hyperspectral (HSI) resolution (1.5 mm GSD) and to the resolution of the enhanced HSI data (0.1 mm GSD). (Wmca-white mica, Bt—biotite, Chl—chlorite, and Gp-gypsum).

After we trained the supervised machine learning model as mentioned in Sections 2.3 and 3.2, we upscaled the high-resolution mineralogical data from the resampled SEM-MLA to the entire enhanced drill-core hyperspectral data and obtained high-spatial resolution minerals maps (see the right side of Figure 9). Moreover, we also included the mineral maps 
obtained with the original hyperspectral data to be able to provide a thorough assessment of the improvement in the mapping when spatially enhancing the hyperspectral data (see the mineral maps on the left side of Figure 9). In general, the high-spatial resolution maps show a visually striking refinement of the structural patterns in the entire samples; for example, the linear features inside the white mica mapped on the left side of Sample 1 are barely seen in the map at original resolution. Complete veins are now visible in the high-spatial resolution maps, as can be observed in Sample 2 with the white mica-chlorite veins mapped in the middle of the sample. In Sample 3, although the main veins are already visible in the map obtained at the original resolution, a great improvement is evident in the matrix, which now appears to be better resolved.

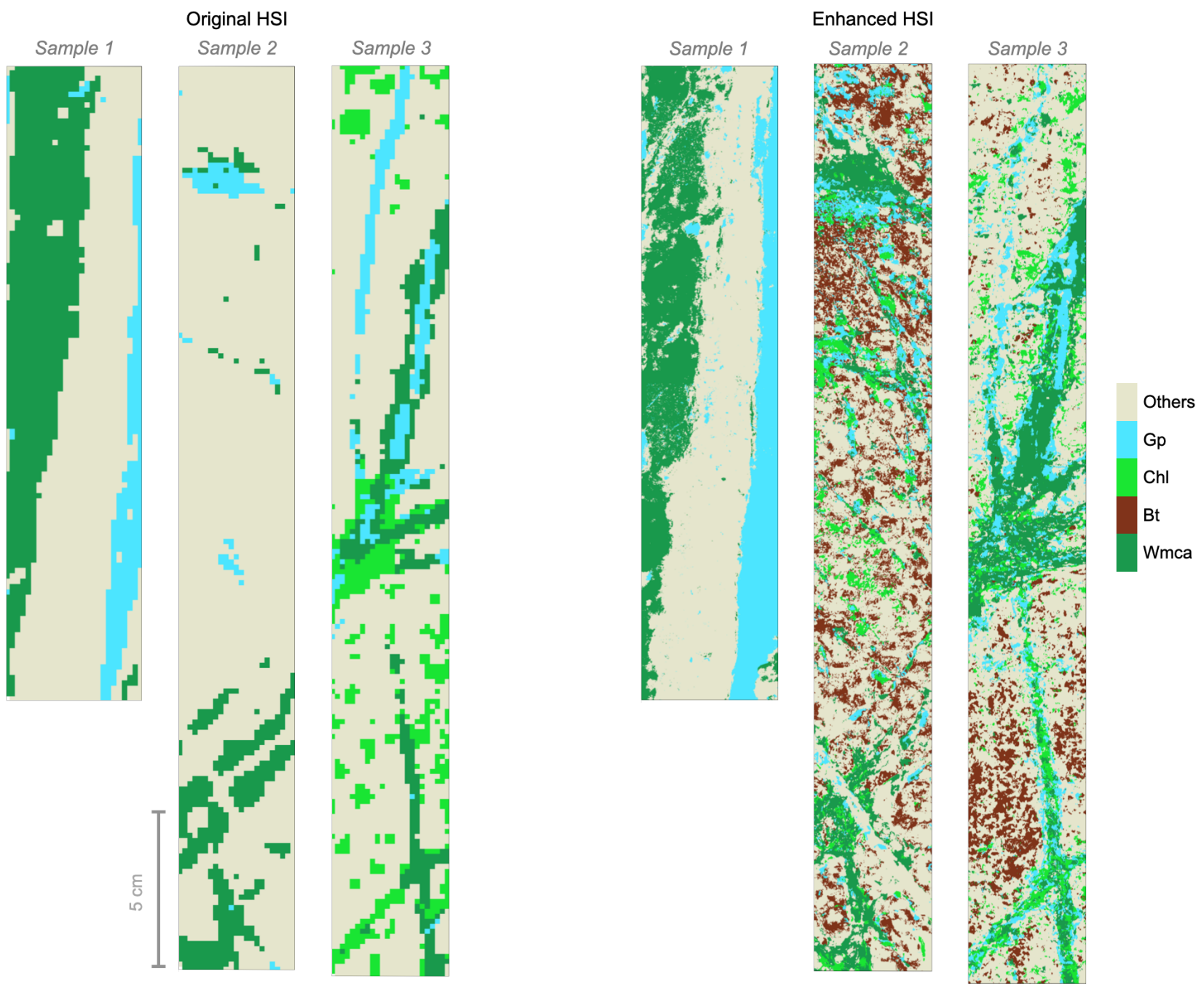

Figure 9. Classified maps obtained by selecting training samples from the co-registered and resampled scanning electron microscopy - mineral liberation analysis (SEM-MLA) data and by classifying the enhanced hyperspectral (HSI) data on the right side with a sampling of $0.1 \mathrm{~mm}$ ground sampling distance (GSD). As a comparison of the proposed method, we also present the classified maps obtained when using the original hyperspectral data (with a sampling of $1.5 \mathrm{~mm}$ GSD) on the left side. (Wmca—white mica, Bt—biotite, Chl—chlorite, and Gp-gypsum).

We visually compared the high-spatial resolution classified maps obtained with the original SEM-MLA data to evaluate and validate the significance of the results (see Figure 2). Overall, the distribution and mapping of the identified minerals agree with the highresolution mineralogical data. For example, we depicted the $G p$ (gypsum) vein on the right side of Sample 1 and the following thick vein mapped with the class Others, which based on the original SEM-MLA corresponds to pyrite that does not have diagnostic absorption 
features in the VNIR-SWIR wavelength range. This thick vein classified as Others is then followed by a Wmca (white mica) region with disseminated gypsum. Similarly, the mapping in Sample 2 is in agreement with the SEM-MLA data. The majority of the veins are a mixture of white mica with less chlorite, and the matrix is mainly mapped as Others and biotite. The mapping in the matrix of Sample 3, however, seems to overestimate the content of biotite. Veins in this sample are correctly mapped as a mixture of white mica, chlorite, and gypsum in certain regions. When comparing the veins in Sample 3 with the SEM-MLA data (see Figure 2), it reveals that the top vertical gypsum vein is better mapped at the original resolution of the hyperspectral data than at the enhanced resolution. However, when looking carefully at the high-resolution mineralogical maps, it is evident that this is a thin gypsum vein with a quartz alteration halo, which is in fact correctly mapped at the enhanced resolution with the class Others. This is also the case of the vein cutting through the white mica veins at the bottom of Sample 2.

\subsection{Validation}

We calculated the accuracies for each of the samples to evaluate the performance of the CCF classifier (see Table 1). In general and as we expected, accuracies are relatively low when using the enhanced hyperspectral data and ranging from about $49 \%$ for Sample 2 to $81 \%$ for Sample 3. Values of the average accuracy seem to be higher than the values of the overall accuracy, which is expected due to the difference in the number of samples per class. Accuracies obtained at the original sampling of the hyperspectral data are higher than those obtained on the enhanced data. However, the comparison and evaluation of the improvement of the mapping at the enhanced sampling using this traditional metric is not appropriate. To be able to evaluate the improvement in the mapping quality due to the increase in resolution, we performed a quantitative analysis over the SEM-MLA resampled maps. We first resized the resampled SEM-MLA data to the original size of the SEM-MLA images and randomly selected $80 \%$ of the total amount of available pixels for each mineral in the original SEM-MLA data and compared those pixels with the corresponding pixels at both the resampled to the original and enhanced hyperspectral resolutions (GSDs of 1.5 and $0.1 \mathrm{~mm}$, respectively). We performed the analysis 100 times and averaged the results. The quantitative analysis shows that the SEM-MLA map resampled to the resolution of the enhanced hyperspectral data preserves the mineralogical information from the original SEM-MLA data in about $98 \%$ and $54 \%$ better than the SEM-MLA data resampled to the original hyperspectral resolution. Figure 10 summarizes this quantitative analysis for the seven most abundant minerals. The opaque bars correspond to the resampled SEM-MLA data at the enhanced resolution, and the transparent bars correspond to the resampled data at the original hyperspectral resolution. In general, the map at the enhanced resolution correctly identified more minerals than the map at the original resolution of the hyperspectral data except for $P l$ for which the difference is very subtle. This figure also highlights how some minerals, for example, $K f s$, $C h l$, and $P y$, are now seen in the SEM-MLA data resampled to the enhanced resolution of the hyperspectral data, whereas at the resolution of the original hyperspectral data, they are lost.

Table 1. Classification accuracies for the canonical correlation forest (CCF) algorithm (\%).

\begin{tabular}{ccccccc}
\hline \multirow{2}{*}{ CCF } & \multicolumn{3}{c}{ Enhanced HSI } & \multicolumn{3}{c}{ Original } \\
\cline { 2 - 7 } & $\mathbf{1}$ & $\mathbf{2}$ & $\mathbf{3}$ & $\mathbf{1}$ & $\mathbf{2}$ & $\mathbf{3}$ \\
\hline Overall & 50.84 & 48.95 & 80.98 & 65.64 & 82.17 & 82.86 \\
Average & 63.17 & 63.41 & 86.78 & 70.24 & 78.37 & 90.67 \\
Kappa & 0.24 & 0.24 & 0.58 & 0.34 & 0.44 & 0.66 \\
\hline
\end{tabular}




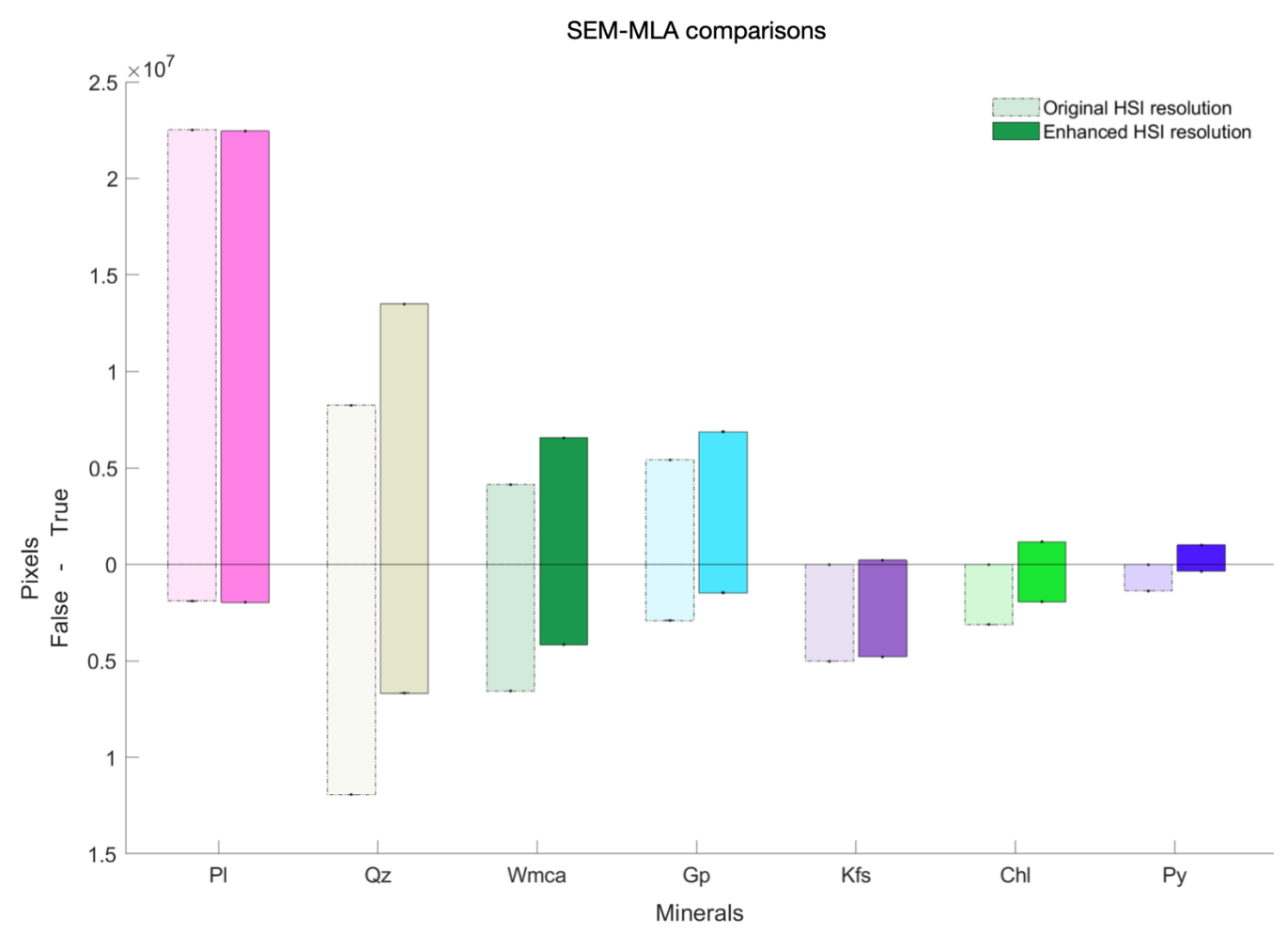

Figure 10. Comparison of mineral identification amongst original scanning electron microscopymineral liberation analysis (SEM-MLA), resampled SEM-MLA at the original resolution of the hyperspectral (HSI) data (dashed lines, transparent bars), and the resampled map at the enhanced resolution (continuous lines, opaque bars). (Pl—plagioclase, Qz-quartz, Wmca-white mica, Gpgypsum, Kfs-K-feldspar, Chl—chlorite, and Py-pyrite).

\section{Discussion}

In this work, we propose a methodology to map minerals in drill-core hyperspectral data at a high-spatial resolution by fusing hyperspectral and high-spatial resolution RGB data. We propose the use of the established CNMF algorithm, which is a subspace-based resolution enhancement technique. We performed a sensitivity analysis over 200 randomly selected pixels to evaluate the spectral integrity after the enhancement process. This sensitivity analysis provides a direct assessment of the impact in the spectra of the resolution enhancement process and evaluates whether the spectra have been preserved or artifacts were added. For this, we used various enhanced hyperspectral datasets obtained with different resolution enhancement factors. The RGB images used for the enhancement of these datasets were generated from the original RGB after a down-sampling step and not acquired with different RGB cameras at different spatial samplings. This sensitivity analysis shows that the spectra are well preserved and that only a general change in the reflectance intensity is observed, which could be due to the involvement of the abundance matrix derived from the RGB data in the process of generating the enhanced data. The fact that the RGB data only have three spectral channels in the visible range of the electromagnetic spectrum (blue, green, and red) has an impact on the general reflectance intensity. This is because the fusion of hyperspectral and multispectral data is usually an ill-posed problem, and when the difference in the spatial sampling of both data sets is large and RGB data (three spectral channels) are used as the multispectral data, unmixing of the RGB data results in a severely ill-posed problem.

The preservation of the spectra is highlighted by the mostly constant values in the spectral similarity measure results. We expected a slight decrease in the measure when using the EF of 15 since this is the one obtained from the original RGB data. The evaluation of the spectral preservation is also possible by looking at the characteristics of the absorption 
features (wavelength position and depth) with the minimum wavelength maps (Figure 6). These initial mineral maps preserve the spatial coherence and do not show any random mapping. The high-spatial resolution minimum wavelength maps clearly highlight the advantages and impact of the enhancement when mapping minerals by better depicting the mineral distribution patterns and showing narrower and more delineated veins. This difference in the mapped veins is highlighted, for example, in the first vertical vein at the bottom left part of Sample 3 at the original resolution. In the map derived from the enhanced HSI, this vein is not mapped as intense as with the original HSI. This is due to the refinement of the spatial distribution of the different spectrally distinct mineral phases during the unmixing process involved in the resolution enhancement step since the presence of white mica is what accentuates this vein in the original HSI.

In this work, we also integrated the enhanced hyperspectral data with SEM-MLA data to map minerals in a supervised manner. The SEM-MLA maps, resampled at both the original and the enhanced hyperspectral resolutions, show that the enhancement allows for the identification and mapping of minerals that are hidden in the original hyperspectral data. For example, the disseminated biotite and chlorite are mapped in the matrix of the SEM-MLA map at the enhanced sampling (see Figure 7). Unraveling the spectra of the objects mapped at the enhanced resolution is, however, still dependent on the scale of the mixtures. Although CNMF operates on a subspace of dimensions given by the number of endmembers determined in the unmixing process within the resolution enhancement, if the intrinsic mixtures of minerals in the sample are at a finer scale than that resolved by the fusion of the RGB and hyperspectral data, mixtures are still observed at the enhanced resolution.

The resolution enhancement step in the supervised mapping of drill-core hyperspectral data not only helps to obtain greater details of the matrix and veins in the cores but also makes it possible to achieve a more explicit identification of the minerals present in the samples. This is because each pixel is assigned a single label when using a hard classifier such as CCF and, therefore, minor components are lost in the mapping. With the increase in GSD in the hyperspectral data, these minor components can now be mapped. This can be observed in the mineral maps we obtained with our proposed supervised mapping (see Figure 9). The use of the extra class Others avoids overestimation of the minerals with a diagnostic response in the VNIR-SWIR and allows for a more accurate representation of the alteration patterns. If hyperspectral data in the long-wave infrared region of the electromagnetic spectrum are available for these samples, it would be possible to fuse the almost full range and, therefore, all of the minerals identified in the SEM-MLA analysis could be used instead of the class Others. This supervised mapping is highly influenced by the quality of the co-registration between the SEM-MLA and the hyperspectral data. To simplify the co-registration, regions where thin sections for SEM-MLA analysis are performed could be marked in the drill-core samples before acquiring the hyperspectral data. In another strategy, the SEM-MLA could be performed in thick sections that can be scanned again with the hyperspectral sensors after removing the carbon coating.

Finally, we performed an accuracy assessment to evaluate the performance of the CCF ensemble classifier when mapping at the original and enhanced resolutions. We built the validation set per class using a number equal to $20 \%$ of the total amount of samples in the smallest class. We expected low values for the accuracies due to the configuration of the validation set. These accuracies, however, demonstrate the good performance of the CCF algorithm, which can be explained by the fact that CCF is an algorithm that performs a split between the classes after being implemented in the feature projections found by the canonical correlation analysis. The higher values of the accuracy when using the original hyperspectral data could be due to the fact that original data are highly mixed, and although we performed an enhancement that involves several unmixing steps, this resultant enhancement is basically spatial and sharpens the mapping, meaning that we increased the number of pixels but some of the obtained pixels still remain mixed. Therefore, in the enhanced data, the number of mixed pixels increases in comparison to 
the original data, causing a small drop in the accuracies since the calculation is performed for their respective resampled training data. However, a direct comparison of the two classifications based on merely accuracies is not convenient since each of them have their specific training and validation sets. The quantitative analysis we performed to evaluate the improvement of the mapping quality highlights how that the data enhancement step aids in reducing the gap between the difference in resolution in both SEM-MLA and hyperspectral data. This in turn allows for preserving the high-resolution mineralogical information from the original SEM-MLA data with an average percentage of 98 as presented in Section 3.2 and Figure 10. The enhancement helps improve the quality of the mapping also due to the better training data; for example, linear features are better delineated at the enhanced resolution, as can be seen with the mapping and refinement of the thin veins in Sample 1 and Sample 2 (see Figure 8).

Implementing resolution enhancement techniques to map minerals in drill-core hyperspectral data allows for the identification of minerals and spatial patterns with hyperspectral data at a higher spatial resolution than traditional mineral mapping techniques performed over the original hyperspectral data. Although the most relevant factor for mineral detection over drill-cores is the spectral quality, including detailed spatial information is highly relevant to better delineate veins, alteration halos, and mineral grains. Having a detailed mapping of such structures enables, for example, the definition of proxies towards mineralization by using the vein composition and orientation and by assessing the stage of alteration. This work was showcased over three drill-core samples; however, the technique can be applied to large datasets as long as the data are co-registered and sufficient storage is available. It is important to highlight storage as the data volume considerably increases when enhancing the spatial resolution of hyperspectral data. As an example, hyperspectral data of 103 bands for 1 meter of drill-core consists of $73 \times 887$ pixels and takes about $22 \mathrm{MB}$. The enhanced hyperspectral data of such sample consists of $511 \times 6209$ pixels and takes around 4.8 GB. This implies that, for an operational environment where, for example, $5 \mathrm{~km}$ of cores are drilled, an average of 24 TB is required for storage. However, the resolution enhancement could be used as an intermediate step and only the subsequent mineral/classified maps or abundance estimates at high-spatial resolution could be stored, considerably reducing the amount of data.

\section{Conclusions}

In this work, we presented a high-spatial resolution mineral mapping approach for drill-core hyperspectral data. We proposed performing an enhancement of the drill-core hyperspectral data using the coupled non-negative matrix factorization (CNMF) algorithm together with high-spatial resolution RGB data. The initial mineral maps highlight that improving the spatial sampling of the hyperspectral data results in a better distribution of the mineral patterns and better delineation of the depicted features, such as veins and mineral grains.

We combined the high-spatial resolution hyperspectral data with high-resolution mineralogical data obtained with the scanning electron microscopy-based mineral liberation analysis (SEM-MLA) to fully explore the possibilities of the resolution enhancement and map minerals in a supervised manner. The enhancement reduces the gap in the spatial sampling between the SEM-MLA and hyperspectral data and allows for a more meaningful synergy between both datasets. Our obtained results show that the mapping not only improved spatially by allowing for the identification of more structures and details (i.e., veins and mineral grains in the matrix of the samples) but also allowed mapping minerals that are hidden at the original resolution of the hyperspectral data. The use of SEM-MLA analysis as reference or training data for a high-spatial resolution supervised characterization of the drill-core hyperspectral data represents a valuable tool for a more automatic upscaling of validated mineralogical information and is beneficial for mapping regions of interest with high detail. This resolution enhancement approach improves the 
mapping of minerals in drill-core hyperspectral data to a level of detail that is favorable in supporting mining companies in their decision-making.

The implementation of the resolution enhancement approach on larger sections such as full bore-holes can be challenging due to the relevant increment in the size of the enhanced data. Additionally, it has been shown in the literature that the performance of the resolution enhancement algorithms reduces once the data is linearly transformed or the dimensionality is reduced, especially when fusing hyperspectral with RGB data (three spectral channels).

In future research, we would like to investigate in detail the impact of more advanced machine learning classifiers not only in mapping but also in the accuracies. Moreover, we will investigate the performance of resolution enhancement algorithms for mineral mapping when fusing hyperspectral data covering the electromagnetic spectrum from the visible-near infrared to the long-wave infrared spectra where more minerals can be identified.

Author Contributions: Conceptualization, I.C.C.A., M.K., and R.G.; methodology, I.C.C.A., M.K., and R.G.; software, I.C.C.A.; validation, I.C.C.A., M.K., and R.G.; formal analysis, I.C.C.A., M.K., and R.G.; investigation, I.C.C.A.; data curation, I.C.C.A.; writing-original draft preparation, I.C.C.A.; writing-review and editing, I.C.C.A., M.K., and R.G.; visualization, I.C.C.A. and R.G.; supervision, M.K. and R.G. All authors have read and agreed to the published version of the manuscript.

Funding: This research received no external funding.

Data Availability Statement: The data presented in this study are available on request from the corresponding author. The data are not publicly available due to restrictions.

Acknowledgments: The authors thank Sandra Lorenz and Samuel Thiele from the HelmholtzZentrum Dresden-Rossendorf (HZDR)—Helmholtz Institute Freiberg for Resource Technology (HIF) for providing insights for the evaluation of the resolution enhancement method. Robert Zimmermann from HZDR-HIF and TheiaX, HZDR Innovation $\mathrm{GmbH}$, and Benjamin Melzer from HZDR-HIF are thanked for their support with the acquisition of the hyperspectral data. Laura Tusa from HZDR-HIF and TheiaX,HZDR Innovation GmbH, and Sabine Gilbricht from HZDR-HIF are gratefully thanked for their assistance with the SEM-MLA analysis.

Conflicts of Interest: The authors declare no conflicts of interest.

\section{References}

1. S\&P Global Market Intelligence. World Exploration Trends 2021; Technical Report; S\&P Global Market Intelligence: New York, NY, USA, 2021.

2. Vidal, O.; Goffé, B.; Arndt, N. Metals for a low-carbon society. Nat. Geosci. 2013, 6, 894-896. [CrossRef]

3. Krahenbuhl, G.; Hapugoda, P.; Warren, K.; O'Brien, G. A new method for obtaining detailed mineral information on individual coal particles at the size that they are used in coke making. In Proceedings of the Bowen Basin Symposium, Melbourne, Australia, 27 September-1 October 2015; pp. 35-40.

4. Haldar, S. Exploration Geochemistry. In Mineral Exploration: Principles and Applications; Elsevier Inc.: Oxford, UK, 2013; Chapter 4, pp. 55-71. [CrossRef]

5. Nikonow, W.; Rammlmair, D. Automated mineralogy based on micro-energy-dispersive X-ray fluorescence microscopy ( $\mu$ EDXRF) applied to plutonic rock thin sections in comparison to a mineral liberation analyzer. Geosci. Instrum. Methods Data Syst. 2017, 6, 429-437. [CrossRef]

6. Fandrich, R.; Gu, Y.; Burrows, D.; Moeller, K. Modern SEM-based mineral liberation analysis. Int. J. Miner. Process. 2007, 84, 310-320. [CrossRef]

7. Clark, R.N. Spectroscopy of rocks and minerals, and principles of spectroscopy. In Remote Sensing for the Earth Science: Manual of Remote Sensing; Rencz, A.N., Ed.; John Wiley \& Sons, Inc.: New York, NY, USA, 1999; Volume 3, Chapter 1, pp. 3-58. [CrossRef]

8. Van der Meer, F. Analysis of spectral absorption features in hyperspectral imagery. Int. J. Appl. Earth Obs. Geoinf. 2004, 5, 55-68. [CrossRef]

9. Clark, R.N.; King, T.V.V.; Klejwa, M.; Swayze, G.A.; Vergo, N. High spectral resolution reflectance spectroscopy of minerals. J. Geophys. Res. 1990, 95, 12653. [CrossRef]

10. Van Ruitenbeek, F.J.; Bakker, W.H.; van der Werff, H.M.; Zegers, T.E.; Oosthoek, J.H.; Omer, Z.A.; Marsh, S.H.; van der Meer, F.D. Mapping the wavelength position of deepest absorption features to explore mineral diversity in hyperspectral images. Planet. Space Sci. 2014, 101, 108-117. [CrossRef] 
11. Van der Meer, F.; Kopačková, V.; Koucká, L.; van der Werff, H.M.; van Ruitenbeek, F.J.; Bakker, W.H. Wavelength feature mapping as a proxy to mineral chemistry for investigating geologic systems: An example from the Rodalquilar epithermal system. Int. J. Appl. Earth Obs. Geoinf. 2018, 64, 237-248. [CrossRef]

12. Kruse, F.A.; Bedell, R.L.; Taranik, J.V.; Peppin, W.A.; Weatherbee, O.; Calvin, W.M. Mapping alteration minerals at prospect, outcrop and drill core scales using imaging spectrometry. Int. J. Remote Sens. 2012, 33, 1780-1798. [CrossRef] [PubMed]

13. Acosta, I.C.C.; Khodadadzadeh, M.; Tusa, L.; Ghamisi, P.; Gloaguen, R. A Machine Learning Framework for Drill-Core Mineral Mapping Using Hyperspectral and High-Resolution Mineralogical Data Fusion. IEEE J. Sel. Top. Appl. Earth Obs. Remote Sens. 2019, 12, 4829-4842. [CrossRef]

14. Acosta, I.C.C.; Khodadadzadeh, M.; Tolosana-Delgado, R.; Gloaguen, R. Drill-Core Hyperspectral and Geochemical Data Integration in a Superpixel-Based Machine Learning Framework. IEEE J. Sel. Top. Appl. Earth Obs. Remote Sens. 2020, 13, 4214-4228. [CrossRef]

15. Barker, R.D.; Barker, S.L.; Wilson, S.A.; Stock, E.D. Quantitative Mineral Mapping of Drill Core Surfaces II: Long-Wave Infrared Mineral Characterization Using $\mu$ XRF and Machine Learning. Econ. Geol. 2020, 116, 821-836. [CrossRef]

16. Tuşa, L.; Khodadadzadeh, M.; Contreras, C.; Shahi, K.R.; Fuchs, M.; Gloaguen, R.; Gutzmer, J. Drill-core mineral abundance estimation using hyperspectral and high-resolution mineralogical data. Remote Sens. 2020, 12, 1218. [CrossRef]

17. Rivard, B.; Harris, N.B.; Feng, J.; Dong, T. Inferring total organic carbon and major element geochemical and mineralogical characteristics of shale core from hyperspectral imagery. AAPG Bull. 2018, 102, 2101-2121. [CrossRef]

18. Thomas, C.; Ranchin, T.; Wald, L.; Chanussot, J. Synthesis of multispectral images to high spatial resolution: A critical review of fusion methods based on remote sensing physics. IEEE Trans. Geosci. Remote Sens. 2008, 46, 1301-1312. [CrossRef]

19. Chen, Z.; Pu, H.; Wang, B.; Jiang, G.M. Fusion of hyperspectral and multispectral images: A novel framework based on generalization of pan-sharpening methods. IEEE Geosci. Remote Sens. Lett. 2014, 11, 1418-1422. [CrossRef]

20. Selva, M.; Aiazzi, B.; Butera, F.; Chiarantini, L.; Baronti, S. Hyper-sharpening: A first approach on SIM-GA data. IEEE J. Sel. Top. Appl. Earth Obs. Remote Sens. 2015, 8, 3008-3024. [CrossRef]

21. Yokoya, N.; Grohnfeldt, C.; Chanussot, J. Hyperspectral and Multispectral Data Fusion: A comparative review of the recent literature. IEEE Geosci. Remote Sens. Mag. 2017, 5, 29-56. [CrossRef]

22. Yokoya, N.; Yairi, T.; Iwasaki, A. Coupled nonnegative matrix factorization unmixing for hyperspectral and multispectral data fusion. IEEE Trans. Geosci. Remote Sens. 2012, 50, 528-537. [CrossRef]

23. Simoes, M.; Bioucas-Dias, J.; Almeida, L.B.; Chanussot, J. A convex formulation for hyperspectral image superresolution via subspace-based regularization. IEEE Trans. Geosci. Remote Sens. 2015, 53, 3373-3388. [CrossRef]

24. Wei, Q.; Dobigeon, N.; Tourneret, J.Y.; Bioucas-DIas, J.; Godsill, S. R-FUSE: Robust Fast Fusion of Multiband Images Based on Solving a Sylvester Equation. IEEE Signal Process. Lett. 2016, 23, 1632-1636. [CrossRef]

25. Yokoya, N.; Chan, J.C.W.; Segl, K. Potential of resolution-enhanced hyperspectral data for mineral mapping using simulated EnMAP and Sentinel-2 images. Remote Sens. 2016, 8, 172. [CrossRef]

26. Dian, R.; Li, S.; Sun, B.; Guo, A. Recent advances and new guidelines on hyperspectral and multispectral image fusion. arXiv 2020, arXiv:2008.03426.

27. Thiele, S.; Lorenz, S.; Kirsch, M.; Acosta, I.C.C.; Tusa, L.; Hermann, E.; Möckel, R.; Gloaguen, R. Multi-scale, multi-sensor data integration for automated 3-D geological mapping using hylite. Ore Geol. Rev. 2021, 136, 104252. [CrossRef]

28. Bakker, W.; van Ruitenbeek, F.J.; van der Werff, H.M. Hyperspectral image mapping by automatic color coding of absorption features. In Proceedings of the 7th EARSEL Workshop of the Special Interest group in Imaging Spectroscopy, Edinburgh, UK, 11-13 April 2011; pp. 56-57.

29. Kokaly, R.; Clark, R.; Swayze, G.; Livo, K.; Hoefen, T.; Pearson, N.; Wise, R.; Benzel, W.; Lowers, H.; Driscoll, R.; et al. USGS Spectral Library Version 7: U.S. Geological Survey Data Series 1035; Technical Report; Publication of US Geological Survey: Reston, VA, USA, 2017. [CrossRef]

30. Rainforth, T.; Wood, F. Canonical Correlation Forests. arXiv 2015, arXiv:1507.05444.

31. Xia, J.; Yokoya, N.; Iwasaki, A. Hyperspectral Image Classification with Canonical Correlation Forests. IEEE Trans. Geosci. Remote Sens. 2017, 55, 421-431. [CrossRef]

32. Tusa, L.; Andreani, L.; Khodadadzadeh, M.; Contreras, C.; Ivascanu, P.; Gloaguen, R.; Gutzmer, J. Mineral mapping and vein detection in hyperspectral drill-core scans: Application to porphyry-type mineralization. Minerals 2019, 9, 122. [CrossRef]

33. Jakob, S.; Zimmermann, R.; Gloaguen, R. The Need for Accurate Geometric and Radiometric Corrections of Drone-Borne Hyperspectral Data for Mineral Exploration: MEPHySTo-A Toolbox for Pre-Processing Drone-Borne Hyperspectral Data. Remote Sens. 2017, 9, 88. [CrossRef]

34. Bachmann, K.; Frenzel, M.; Krause, J.; Gutzmer, J. Advanced Identification and Quantification of In-Bearing Minerals by Scanning Electron Microscope-Based Image Analysis. Microsc. Microanal. 2017, 23, 527-537. [CrossRef]

35. Kern, M.; Möckel, R.; Krause, J.; Teichmann, J.; Gutzmer, J. Calculating the deportment of a fine-grained and compositionally complex Sn skarn with a modified approach for automated mineralogy. Miner. Eng. 2018, 116, 213-225. [CrossRef] 
36. Chang, C.I.; Du, Q. Estimation of Number of Spectrally Distinct Signal Sources in Hyperspectral Imagery. IEEE Trans. Geosci. Remote Sens. 2004, 42, 608-619. [CrossRef]

37. Kruse, F.A.; Lefkoff, A.B.; Boardman, J.W.; Heidebrecht, K.B.; Shapiro, A.T.; Barloon, P.J.; Goetz, A.F. The spectral image processing system (SIPS)-interactive visualization and analysis of imaging spectrometer data. Remote Sens. Environ. 1993, 44, 145-163. [CrossRef] 